\title{
Corela
}

Cognition, représentation, langage

HS-4 | 2006

Le parcours

\section{Stabilité et qualification dans les WH dits de parcours}

Régis Mauroy

\section{CpenEdition}

Journals

Édition électronique

URL : http://journals.openedition.org/corela/1387

DOI : $10.4000 /$ corela. 1387

ISSN : 1638-573X

Éditeur

Cercle linguistique du Centre et de l'Ouest - CerLICO

Référence électronique

Régis Mauroy, «Stabilité et qualification dans les WH dits de parcours », Corela [En ligne], HS-4 | 2006, mis en ligne le 08 juin 2006, consulté le 20 avril 2019. URL : http://journals.openedition.org/ corela/1387 ; DOI : 10.4000/corela.1387

Ce document a été généré automatiquement le 20 avril 2019

\section{(c) (i) (2)(2)}

Corela - cognition, représentation, langage est mis à disposition selon les termes de la licence Creative Commons Attribution - Pas d'Utilisation Commerciale - Partage dans les Mêmes Conditions 4.0 International. 


\title{
Stabilité et qualification dans les WH dits de parcours
}

\author{
Régis Mauroy
}

\section{0 . Introduction}

1 Cet exposé fait suite à une étude portant sur les WH en anglais (Mauroy 2003) et vise à préciser ce qui nous semble être la nature fondamentale des opérations communes à cette classe de marqueurs dans TOE. Nous ferons ainsi tout d'abord quelques remarques sur l'hétérogénéité de fait observable à propos de ces différents marqueurs, et d'autre part, chercherons à identifier les propriétés qui leur sont communes. Nous verrons alors que l'opération dite de parcours, souvent utilisée pour décrire l'opération WH, ne peut pas être décrite directement en termes de parcours d'occurrences d'une classe ouverte, paradigme auquel renverrait le marqueur, mais plutôt en fonction du degré de stabilité et de non-stabilité de la composante qualitative qui lui est attachée.

\section{Parcours interrogatif et $\mathrm{WH}$ relatif}

2 Les marqueurs en WH dans leur ensemble sont, on le sait, foncièrement des interrogatifs ${ }^{1}$ : par leur étymologie tout d'abord, et c'est sans doute la seule propriété énonciative ou syntaxique qu'ils possèdent effectivement en commun (à l'exception de whether pour l'interrogation directe, mais seulement indirecte), mais en fait le seul trait d'homogénéité dans une classe par ailleurs très hétérogène, ainsi qu'en atteste le tableau suivant :

\begin{tabular}{|l|l|l|l|l|l|l|}
\hline & $\begin{array}{l}\text { Qlt } \\
\text { uniquement }\end{array}$ & $\begin{array}{l}\text { Qlt, } \\
\text { Qnt }\end{array}$ & $\begin{array}{l}\text { Adverbial } \\
\text { Qlt-Qnt }\end{array}$ & $\begin{array}{l}\text { Relatif sans } \\
\text { antécédent }\end{array}$ & $\begin{array}{l}\text { Relatif avec } \\
\text { antécédent }\end{array}$ & Conjonction \\
\hline Who & & $\mathrm{X}$ & & possible (1) & $\mathrm{X}$ & \\
\hline
\end{tabular}




\begin{tabular}{|c|c|c|c|c|c|c|}
\hline Whom & & $x$ & & possible (1) & X & \\
\hline Whose & & $x$ & & possible (2) & X & \\
\hline What & & $x$ & & $\mathrm{x}$ & $\begin{array}{l}\text { impossible * } \\
\text { (dialectal) }\end{array}$ & \\
\hline Which & & $\mathrm{X}$ & & $\begin{array}{l}\text { possible (3) } \\
\text { (rare) }\end{array}$ & X & \\
\hline When & & & $\mathrm{X}$ & $\mathrm{X}$ & $\mathrm{X}$ & $\mathrm{x}$ \\
\hline Where & & & $\mathrm{x}$ & $\mathrm{x}$ & X & $\mathrm{x}$ \\
\hline Why & & & $\mathrm{X}$ & $\mathrm{x}$ & $\mathrm{X}$ & impossible \\
\hline How & $\mathrm{X}$ & & possible & $\mathrm{X}$ & impossible* & impossible \\
\hline Whether & & $x$ & $\mathrm{X}$ & $\mathrm{x}$ & $\mathrm{X}$ & $\mathrm{X}$ \\
\hline ... & & & & & & \\
\hline
\end{tabular}

* Seuls what et how semblent impossibles comme relatifs avec un antécédent.

4 On remarque par ailleurs que ce sont les deux seuls WH qui marquent une classe totalement ouverte : how sur le plan qualitatif et what sur le plan qualitatif et quantitatif.

(1) 'She went out with who(m) you know.'

(2) 'I'l tell you whose car you can borrow.'

(3)'There was Paul and John, but I know which (of them) you spoke to.'

5 Notons tout d'abord qu'un énoncé interrogatif en WH (tout comme une yes/no-question) est bien stabilisé au sens où il est évidemment chaque fois interprétable, mais uniquement dans le cadre de la modalité inter-sujets impliquant à la fois l'impossibilité d'une référenciation et donc d'une validation immédiate, mais différée et remise à l'initiative du co-énonciateur. Il s'agit de ce que l'on peut appeler une issue sur le plan énonciatif à l'instabilité primordiale instaurée par les marques d'un déficit référentiel.

Or, une question nous paraît fondamentale dans l'étude de cette classe de marqueurs hétérogènes: pourquoi certains d'entre eux sont-ils employés comme relatifs, avec antécédent (which, who, where, when, why, et même parfois whether) alors que d'autres (singulièrement what et how) ne connaissent pas cet emploi, du moins en anglais standard contemporain et sont alors parfois décrits comme "relatifs sans antécédent", ou "à antécédent interne" dans ce qu'il est convenu d'appeler les propositions relatives nominales ? L'opération de parcours ne semblerait donc pas être fondamentalement liée à leur fonctionnement. Il nous paraît donc souhaitable d'examiner s'il est indispensable de poser ce type d'opération pour rendre compte du fonctionnement de l'ensemble de la classe des WH et de leurs emplois et fonction diverses. 


\section{Deux opérations fondamentales}

7 Nous définirons en fait deux opérations fondamentales et complémentaires, communes à tous les WH de la manière suivante :

$1^{\circ}$ ) La marque d'une absence référentielle autonome : place "vide" ou "vidée", à la

fois lorsqu'ils sont interrogatifs et relatifs, c'est-à-dire anaphoriques).

C'est alors le choix du marqueur en WH qui indique la nature de la place ainsi vidée dans la RP : argument (non-animé ou animé humain) ou circonstanciel pour les catégories de temps, lieu, cause, manière, etc. La place syntaxique est par ailleurs peu pertinente puisque l'interrogation et la construction relative à un moindre degré placent ce terme en tête de la prédication.

$\left.2^{\circ}\right)$ La mise en place d'une relation de repérage inter-propositionnelle découlant directement de ce vide référentiel.

9 Dans l'interrogation directe, cette relation s'instaure dans l'intersubjectivité liée à ce type d'assertion. Pour l'interrogation indirecte, elle s'établit sous la forme d'un repérage syntaxique ainsi que pour les relatifs (avec identification anaphorique dans le cas des relatifs renvoyant à un antécédent). Enfin, c'est cette même relation qui donne lieu à une boucle d'identification dans les énoncés exclamatifs.

10 Il est important de souligner pourquoi ces deux opérations fondamentales sont à la fois distinctes et parfaitement complémentaires. Une place vide marquant une impossibilité référentielle totale ne peut être imaginée dans le processus énonciatif visant à lever de l'indétermination. Même un constat brut du type : 'I dont know' peut dans certains cas être interprété comme une question et donc un recours à autrui. Comme pour les anaphoriques qui en soi ne sont pas interprétables et s'identifient à un interprétant, une place vide ne peut, de manière générale, être destinée qu'à construire une opération de repérage de manière à différer l'interprétation.

11 C'est donc en fonction de ces deux paramètres de base que nous voulons analyser l'opération de parcours souvent prêtée aux WH lorsqu'ils marquent effectivement l'indétermination (ou la non-détermination).

\section{Parcours et domaine}

12 Dans une étude précédente (Mauroy, 2003), nous avancions déjà l'idée selon laquelle un parcours des valeurs instanciables sur un domaine n'est sans doute pas la façon la plus adéquate de décrire WH. En effet, il faut d'abord examiner la façon dont est construit un tel domaine notionnel. Tout d'abord, il ne peut s'agir d'un parcours d'occurrences construites et repérées dans un domaine à moins qu'il n'existe une opération de nature anaphorique distincte des WH (ce qu'ils ne sont pas naturellement) avec which, par exemple. Par ailleurs, le parcours d'un domaine notionnel tel qu'on peut le décrire pour les marqueurs any ou every par exemple (et beaucoup d'autres) est fondamentalement référentiel, au sens où les occurrences considérées sont rendues quantifiables dans un domaine et parcourues sur cette base. Cette dimension quantitative est présente chez certains WH par l'existence de propriétés complémentaires des marqueurs portant sur le type de référence (who et what par exemple : classe des animés humains/classe des non animés, dont toutes les occurrences sont quantifiables). Mais à l'opposé, ce n'est pas le cas 
pour how qui n'appelle que des propriétés strictement qualitatives, même si des opérations énonciatives peuvent ensuite construire le quantitatif de différentes manières: repérage d'occurrences distinctes répondant au vide informationnel, prédications situées, etc.

Il nous semble donc qu'il ne faille pas analyser l'ensemble des WH sur la base de certains des marqueurs seulement puisqu'ils présentent chacun des opérations complexes, et que pour l'ensemble de la classe, il s'agit plutôt d'une prise en compte des propriétés qualitatives définissant un domaine ouvert.

14 A ce stade, notre analyse n'était pas axée sur la notion de parcours et nous avions davantage privilégié la distinction entre composante qualitative notionnelle et différentielle, l'étude des pré-construits, ainsi que l'orientation des opérations de repérage. Il faut ajouter à cela le critère de stabilité (ou de non-stabilité) de ces propriétés en fonction de la lexis non saturée à laquelle renvoient de telles prédications.

\section{Place vidée et composante qualitative dans I'interrogation} type : <He - like ( )>. Elle n'est donc pas "saturée". Mais un telle place vide d'argument n'est pas instanciable dans un énoncé nécessitant les opérations énonciatives préalables à la référenciaton. C'est à la faveur tout d'abord de la quantification de cette lexis en une occurrence repérée, puis de son instanciation par les opérations de détermination que peut apparaître un marqueur tel que what renvoyant à une place "vidée" de toute valeur instanciable par l'énonciateur. Les déterminations, temporelles, aspectuelles, et notamment modales construisent un énoncé interrogatif portant sur cette valeur (instanciable, mais non instanciée), à la fois du point de vue qualitatif et quantitatif. On obtient ainsi une question dite ouverte: toute valeur envisageable peut a priori être attribuée à cet argument dans un énoncé asserté.

19 Toute lexis construit une notion complexe, composée de termes et de relations lui fournissant des propriétés spécifiques, et donc différentielles de tout autre lexis. Ces arguments instanciés ainsi que ces relations fournissent ainsi une notion complète pour une lexis saturée et incomplète pour une lexis non saturée pour laquelle une notion complexe existe néanmoins. Le marqueur en $\mathrm{WH}$ marque donc la présence d'une valeur qualitative différentielle (Qlt différentiel) sans la fournir, puisque vide de propriétés spécifiques. On peut dire que celle-ci n'est certes pas explicite, fournie en extension par le marqueur, mais au contraire en intension par la lexis. C'est pourquoi, par ailleurs, un énoncé interrogatif comportant plus d'un seul marqueur en WH n'est interprétable que 
dans certaines conditions (en dehors de la difficulté syntaxique à placer les deux interrogatifs en tête d'énoncé) :

* What does who like?

Who does what?/Who likes what?

qui peut être complété :

Who does what (where, when and why...)?

Les exemples suivants en attestent :

(5) Moscow authorities are making it quite clear they'll decide who does what in the city and not the Kremlin. (BNC KRT 5312)

(6) It enables departmental 'top management' to ascertain within their departments who does what, why and at what cost. (BNC G19 85)

(7) A foolproof guide to who does what, where, when and how. (BNC BPF 277)

Quel rôle qualitatif peut-on dès lors prêter à WH? Ne marque-t-il que l'absence et donc le vide notionnel? Ces questions se posent avec davantage d'acuité quand il s'agit des interrogatives, en raison de la part d'indétermination évidente qui règne en raison de l'absence référentielle ('What does he like ?'). La composante qualitative n'est certes pas spécifiée dans ce cas, mais tire indéniablement tout un ensemble de propriétés fournies par la relation elle-même et les termes instaurés par la lexis. Il ne peut s'agir que de propriétés notionnelles interprétables en fonction de cette lexis puisqu'elles lui sont associées, les seules propriétés spécifiques, différentielles restant indéterminées mais potentielles. Les occurrences visées sont non stabilisées dans un domaine notionnel ouvert sur la base même de cette lexis.

24 C'est pourquoi WH apparaît également dans le cas d'une référence identifiée dans l'énoncé ou le co-texte (relatives nominales ou pseudo-clivées). Il ne s'agit plus alors de modalité interrogative et d'indétermination et l'on comprend mieux que le qualitatif Qlt de WH puisse être fourni à la fois par la prédication où il s'inscrit et une prédication "déterminante" à laquelle il est lié syntaxiquement selon différentes fonctions. Cette identification qualitative, il est vrai, est tantôt explicite et tantôt implicite :

(8) 'what he likes is a little bit of mystery.'

(9) 'He told me what he likes.'

\section{La préconstruction}

La question de la préconstruction est fondamentale pour aborder le parcours et définir sur quel type de domaine il devrait opérer.

Il apparaît que la lexis sur laquelle opèrent l'interrogation est en effet prise en compte dans sa dimension non saturée marquée par une ou parfois plusieurs places vides, mais qu'elle n'est pas d'emblée préconstruite comme assertée ou même validée sur les termes saturés, impliquant par exemple pour : 'What does he like?', He likes [ ], habituellement glosé par: He likes something. On voit par ailleurs qu'un marqueur tel que something marque l'existence d'opérations qui sont absentes de l'énoncé interrogatif considéré.

Il ne paraît donc pas indispensable pour rendre compte des opérations de présenter le terme désigné par WH ou la RP comme préconstruits. C'est pourtant ce que l'on fait lorsqu'on explique qu'un énoncé du type :

(10) “'What happened to the baby?', she asked." (BNC HGV 3372) 
préconstruit l'énoncé (ou les termes mis en relation de l'énoncé) : < (something) happened to the baby>

En revanche, la préconstruction dans l'interrogation nous semble possible dans les énoncés que nous appelons interrogatives clivées du type :

(11) 'What is it that you want me to do, Isabel?' (BNC AD1 1624) ou encore :

(12) “'What is it that you wish to discuss?', he asked." (BNC ANL 2942)

Une série d'opérations liées au clivage pose alors l'existence d'une occurrence construite mais non stabilisée, sur laquelle porte ensuite l'interrogation (voir Mauroy, 2003).

2 Il est d'ailleurs à remarquer que le terme en WH marque à la fois une composante qlt notionnelle (dont les propriétés proviennent de la lexis), ainsi que l'existence de propriétés qualitatives différentielles qu'il représente comme non stabilisées. Ce type de focalisation fait également du terme focalisé un terme différentiel à l'exclusion de tout autre. On obtient dans ce cas une composante qualitative doublement différentielle.

Dans tous les cas (interrogation ou relative, avec ou sans antécédent) la prédication construit son contenu sans poser de relation ou de terme préconstruit. L'étude de la relative montre bien que s'il y a préconstruction d'une relation, RP, celle-ci est due a d'autres facteurs :

C'est le cas de déterminatives restrictives dont l'antécédent est déterminé par a. Avec préconstruit, on trouve par exemple :

(13) 'This was a man who liked order.' (BNC A8Y 178)

ou sans préconstruit :

(14) 'Currently, most people won't go and see a band who they haven't heard of.'

(BNC A6A 98)

Plutôt que de parcours d'occurrences sur un domaine construit, nous préférerons donc parler pour WH d'une marque de l'hétérogénéité des composantes différentielles d'un domaine notionnel non homogène organisé autour du centre organisateur. Cette hétérogénéité demeure dans l'interrogation grâce au caractère non stable qu'implique nécessairement l'indétermination, mais disparaît après stabilisation, par identification anaphorique par exemple.

37 Les pronoms what / which / who et whom / whose posent d'emblée la nécessité d'une composante quantitative Qnt. La relation entre place vidée et terme référentiel ne peut s'effectuer que sur la base d'occurrences quantifiées et donc situées, mais non pas nécessairement identifiées. La stabilité ou non stabilité du qualitatif différentiel (il y a ou il n'y a pas à ce stade de propriétés différentielles envisageables...) détermine alors si des occurrences sont distinguées.

38 En revanche, pour les marqueurs circonstanciels When, Where, Why, How, dont la composante fondamentale est également qualitative, aucune composante Qnt n'est posée a priori. On voit donc que les propriétés différentielles peuvent être marquées seules et pour elles-mêmes.

Pour la plupart d'entre eux, toute opération de mise en relation n'est possible qu'en fonction de telles propriétés Qlt qui doivent également être quantifiée s'il ne s'agit pas de marqueurs déictiques. 
Pour résumer (et simplifier à ce stade) :

- When ? (déictiques : now/then/today, etc. / SN : one day, etc. / SP : in 2000, etc. / proposition circonstancielle : when ..., etc.)

- Where ? (here/there, etc. / SP : "in the car", etc. / proposition : "where .....")

- Why? (aucun déictique sinon construit : because of this/that / SP : "because of etc. / proposition : because ..., etc.)

- How? (aucun déictique sinon construit : (in) this/that way etc. /SP : "in a [...] way/ manner,etc.", etc. / proposition explicative de la manière). Mais un terme uniquement qualitatif peut éventuellement convenir, par exemple un adverbe non déictique en -ly (propriétés sémantiques lexicales non quantifiées par elle-mêmes) : properly / carefully / etc.) Un terme quantifié peut également être construit.

41 On voit que how peut demeurer purement qualitatif (hors de toute opération subsidiaire de quantification) et qu'il ne peut s'agir fondamentalement que d'un parcours de ces propriétés, et non d'occurrences d'une classe, d'un domaine. Ici encore, le caractère non stable concerne en premier lieu le qualitatif. Toute quantification, par exemple par la construction d'énoncés répondant au vide informationnel, ne peut intervenir que comme une série d'opérations secondes servant à instancier des propriétés fondamentalement qualitatives et différentielles.

\section{Conclusion}

Il n'est pas possible en quelques lignes d'explorer toute la complexité des marqueurs considérés, mais l'examen des opérations premières nous amène à tirer quelques conclusions provisoires.

Parcourir une classe ou un domaine impose de préconstruire cette classe en termes d'occurrences. C'est ce que fait le marqueur which par exemple, par une opération distincte de $\mathrm{WH}, \mathrm{du}$ fait de sa nature systématiquement anaphorique quel que soit son rôle (interrogatif ou relatif), et il opère alors un parcours de ces occurrences sur un domaine préconstruit par le co-texte ou le contexte.

Les marqueurs who et what posent la nécessité d'une composante quantitative sans pour autant construire un domaine susceptible de parcours.

Les circonstanciels when, where, why, how ne posent pas cette nécessité qui s'impose ou non dans la construction ultérieure d'une référence.

Whether pose une lexis au niveau qualitatif dans son rapport avec la validation ou la nonvalidation (bifurcation), c'est-à-dire son appartenance à I ou E du domaine, depuis IE, sans construction d'occurrence de cette lexis a priori.

Toute opération fournissant une stabilité qualitative à WH est seulement susceptible de construire Qnt. Toute opération introduisant au contraire une non-stabilité qualitative ne permet pas cette quantification. 


\section{BIBLIOGRAPHIE}

COLLINS P. Cleft and Pseudo cleft constructions in English, Routledge, London and New York, 1991.

CULIOLI A. (1991-1999) Pour une Linguistique de l'Enonciation, Opérations et représentations, Tome 1, Formalisation et opérations de repérage, Tome 2, Le domaine notionnel, Tome 3, Ophrys, Paris.

DANON BOILEAU L. «This, that, which, what et la construction de références», in Méthodes en Linguistique Anglaise, CIEREC. XXXIX, Université de Saint Etienne, 1983.

FLINTHAM R. «Les relatifs which et that dans un corpus journalistique», in Cahier Charles $\mathrm{V}, \mathrm{n}^{\circ}$ 19, Linguistique et didactique Université de Paris 7, 1995.

FLINTHAM R. «Les relatives en which et that dans Scientific American» in Danon Boileau L. et Duchet J-L. éds, Opérations énonciatives et interprétations de l'énoncé. Mélanges offerts à Janine Bouscaren, 1993, pp. 171-179.

GIRARD G. «Propositions enchâssées en WH- : interrogatives indirectes, relatives nominales, ou troisième type de proposition ?» in Mélanges en l'honneur de Gérard Deléchelle, Groupe de Recherches Anglo-Américaines de l'Université François Rabelais de Tours, Publications de l'Université François Rabelais, Tours, 2001.

GOURNAY L. 2003, « Remarques sur les emplois propositionnels des marqueurs en WH- : une perspective énonciative », communication à la journée sur WH-, Université de Rouen, disponible en ligne sur le site de l'ALAES.

GRESSET S. «Which/That marqueurs de relatives», in Bouscaren J. Cahiers de Recherche en Grammaire Anglaise, n², Gap, Ophrys, 1984.

KHALIFA J.C. La Syntaxe anglaise aux concours CAPES/AGREG : Théorie et pratique de l'énoncé complexe, Paris, A. Colin, 1999.

MAUROY R. (2003) « Quel WH- dans les interrogatives les relatives et les clivées ?» in Interlangues, La subordination en anglais, une approche énonciative, Stéphane Gresset et Agnès Celle Eds, Sous la direction de Wilfrid Rotge, Presses Universitaires du Mirail, Université de Toulouse-Le Mirail.

MAUROY R. (2005, à paraître) «Les énoncés interrogatifs clivés en anglais, le jeu du qualitatif dans les opérations de repérage», in ANGLOHONIA, N¹5, Presses Universitaires du Mirail.

MAUROY R. (2005, en préparation) «How is it that manner means cause ? Comment se fait-il que la manière exprime la cause ?» Travaux du CERLITEP de Poitiers, Causalité, Corpus, Contrastivité, Presses Universitaires de Rennes.

MAUROY R. (2005, en préparation) «La manière en questions » Colloque de Linguistique anglaise La qualification, CRELA, Université de Nice, septembre 2005.

MAUROY R. (2005, en préparation) «HOW connecteur syntaxique : construction qualitative et quantitative de la manière», Colloque Les connecteurs, Institut d'anglais Charles- $\mathrm{V}$, Université Paris-7 Denis Diderot, mai 2005. 
PRINCE E. «A comparison of WH-clefts and it-clefts in discourse», in Language, 54, p 883-906, 1978.

QUIRK R., GREENBAUM, S., LEECH G. \& SVARTVICK J. A Comprehensive Grammar of the English Language, London : Longman, 1985.

REYNAUD C. « Cleft-Sentences Versus Restrictive Relative Clauses in English : A Syntactic and Enunciative Comparison », mémoire de Maîtrise sous la direction de Jean Chuquet, Université de Poitiers, 2001.

\section{NOTES}

1. Je remercie Annie Lancri de m'avoir fait remarqué qu'il existait malgré tout dès le vieilanglais des emplois distincts de la seule interrogation.

\section{RÉSUMÉS}

Les marqueurs anglais en WH, d'origine interrogative, sont souvent décrits dans la Théorie des Opérations Enonciatives comme renvoyant à l'opération de parcours comme s'il s'agissait d'une opération première et constitutive de l'ensemble de ces marqueurs. Nous voulons montrer qu'une analyse générale de cette classe fondamentalement hétérogène ne peut déboucher sur une telle description. Au-delà de leurs différentes fonctions grammaticales et syntaxiques recensées en anglais contemporain, qui varient selon les marqueurs considérés (interrogatifs directs et indirects, relatifs avec ou sans antécédent, etc.), on remarque que les composantes qualitative Qlt et quantitative Qnt ne se situent pas dans le même rapport avec les opérations selon les marqueurs. La seule constante est une place vidée dans la lexis, posant par sa présence une relation de repérage le plus souvent inter-propositionnelle. Cette place vidée est la marque d'une non-stabilité des propriétés différentielles alors que la lexis ainsi repérée fournit par ellemême un ensemble de propriétés notionnelles. Il n'est ainsi pas nécessaire de poser un domaine préconstruit d'occurrences pouvant ensuite être parcourues pour rendre compte des interrogatives ni des relatives simples, à moins que des opérations particulières, de détermination (fléchage) ou de focalisation (clivage) par exemple, ne posent la préconstruction d'un tel domaine.

WH markers in English being originally interrogative are most often described in TOE as referring to scanning, considering that this is a primary operation in all these markers. We will show that a general analysis of this fundamentally heterogeneous class of markers cannot conclude to this type of description. Besides the differences existing in their grammatical and syntactic functions, as they may be observed in the various markers in contemporary English (direct or indirect interrogative and relative pronouns or adverbs with or without an antecedent), it can be noticed that the qualitative and quantitative components Qlt and Qnt, do not play the same part in the operations of each marker. The only common and fundamental operations to be found are the mark of an empty or emptied place in the lexis along with that of a relation locating it relative to another predication. When the former marks the absence of stability of the differential properties involved, the latter provides the notional properties. It is 
therefore unnecessary to conceive a notional domain whose occurrences may be scanned, in order to account for simple interrogative or relative clauses, unless other specific operations of determination (pinpointing) or focalisation (cleft-structures) for instance, do reveal the preconstruction of such domains.

INDEX

Mots-clés : parcours, stabilité, déformabilité, qualification, quantification, interrogation ouverte, pré-construit, repérage inter-prédicationnel, place vide

Keywords : scanning, stability, deformability, qualification, quantification, wh-questions, interpredicational relation, empty slot

\section{AUTEUR}

\section{RÉGIS MAUROY}

Université de Limoges 